\title{
NEUTROPHIL TO LYMPHOCYTE RATIO (NLR) - RELATIONSHIP WITH ANTHROPOMETRIC AND METABOLIC PARAMETERS IN MORBIDLY OBESE PATIENTS
}

\section{Cristina Serbanescu ${ }^{1}$, Liliana Topor ${ }^{1}$, Ivona Gheorghe-Fronea ${ }^{1}$, Alexandra Nila ${ }^{1}$, Anca Sirbu ${ }^{1,2}$, Simona Fica ${ }^{1,2}$}

1. Elias Emergency University Hospital, Bucharest, Romania

2. University of Medicine and Pharmacy „Carol Davila”, Bucharest, Romania

\section{Introduction}

Obesity is generally associated with a chronic state of subclinical inflammation. The assessment of the inflammatory status is routinely made by measuring circulating levels of nonspecific proinflammatory markers. Neutrophil to lymphocyte ratio (NLR) is a new simple inflammatory marker which can be reliable in evaluating the inflammatory status occurring in morbidly obese patients

\section{Patients and methods}

Our study included 415 patients (130 men) with severe obesity (mean $\mathrm{BMI}=45.46 \pm 8.68 \mathrm{~kg} / \mathrm{m}^{2}$, mean age $=41.31 \pm 11.33$ years), who were evaluated clinically (medical history, anthropometrics, blood pressure measurements) and biologically (blood count, complete metabolic tests, leptin level) in a research program for bariatric surgery.

\section{Objectives}

The aim of this study was to investigate the relationship between NLR as an index of chronic inflammation and anthropometric and metabolic parameters in a group of morbidly obese patients.

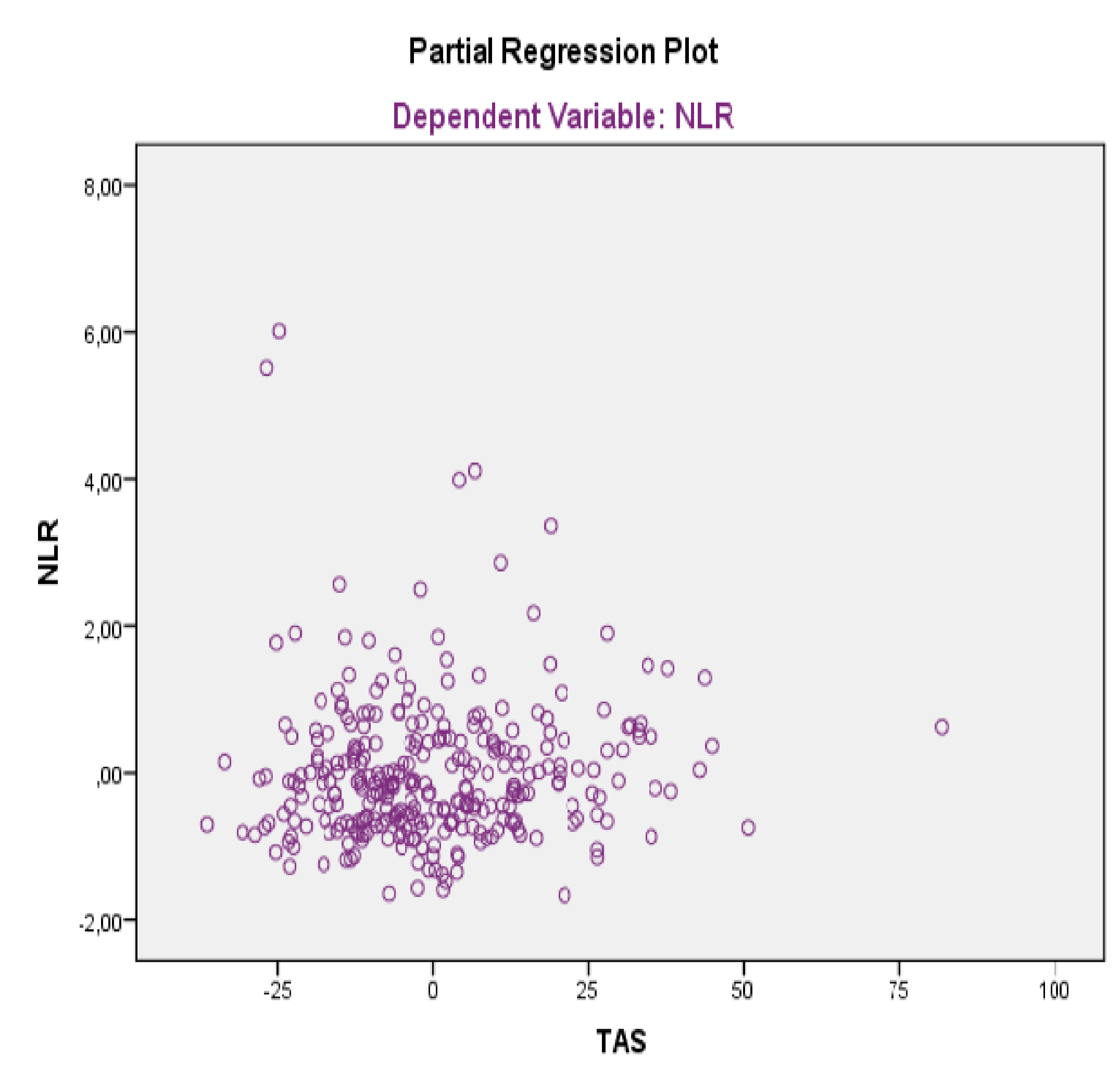

Fig. 3, Relationship between NLR and Systolic blood pressure

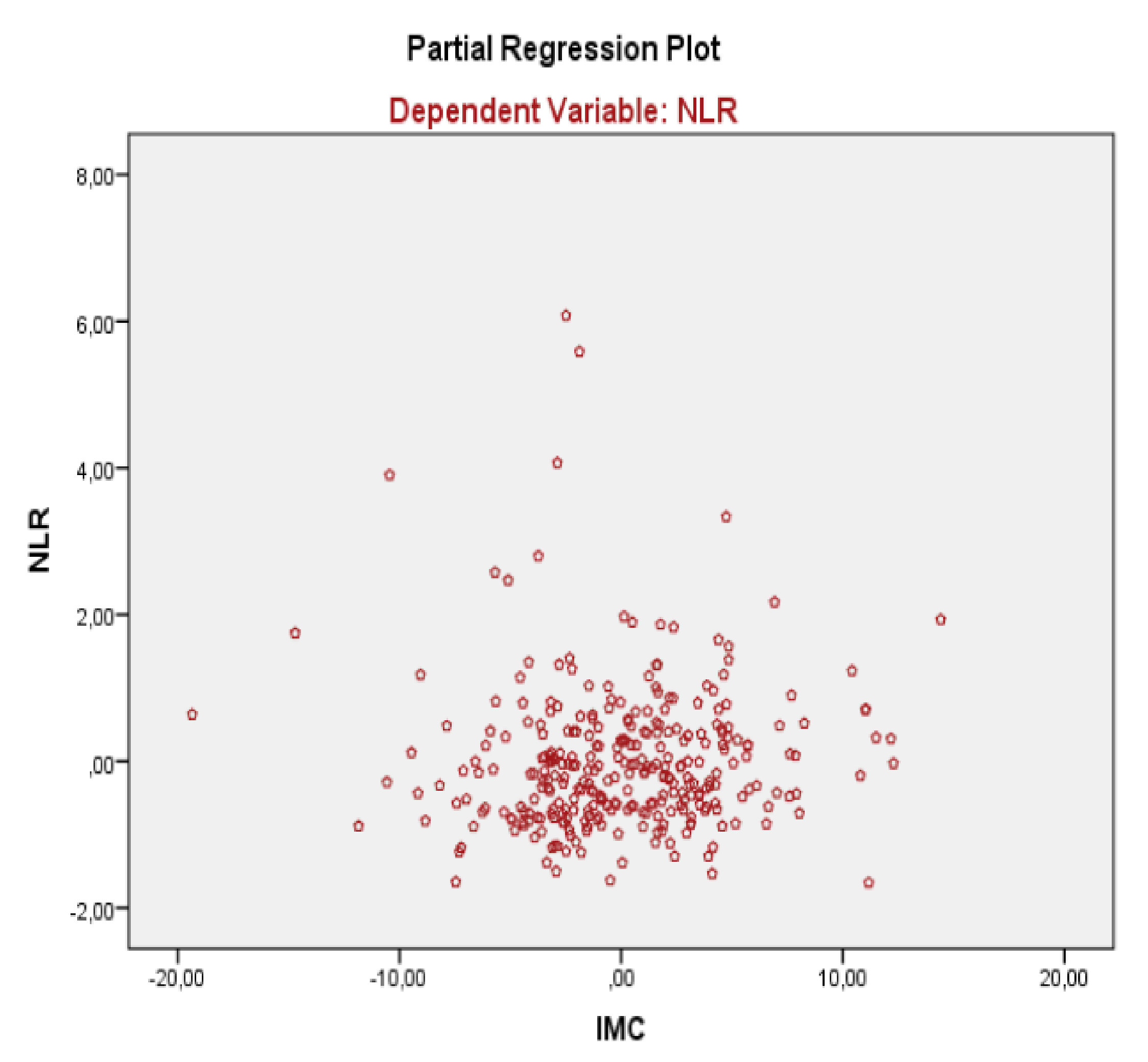

Fig. 4, Relationship between NLR and BMI

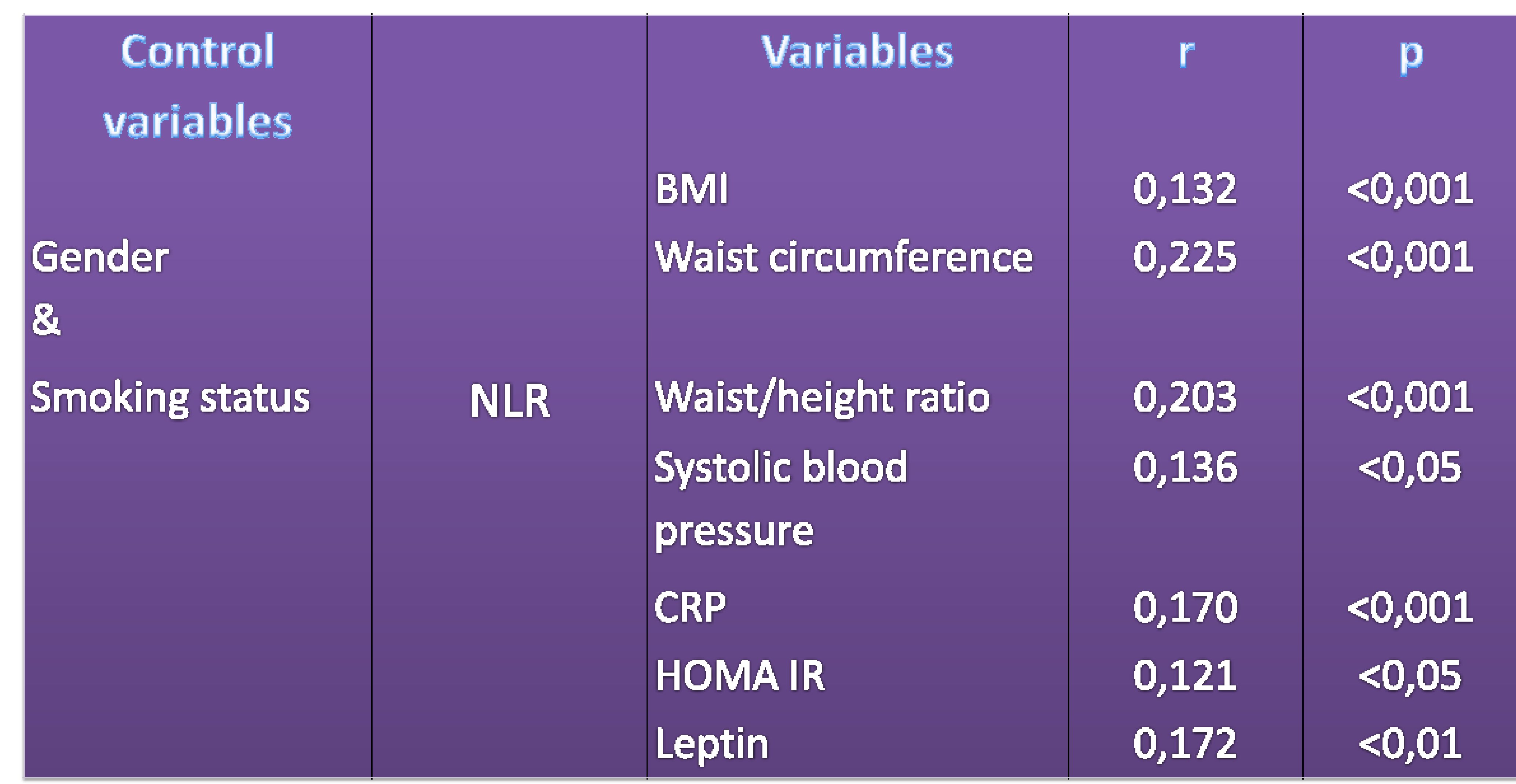

Table 1, Positive correlations between NLR and BMI, Waist, Waist/Height ratio, Systolic $B P, C R P, H O M A I R$, Leptin

Partial Regression Plot

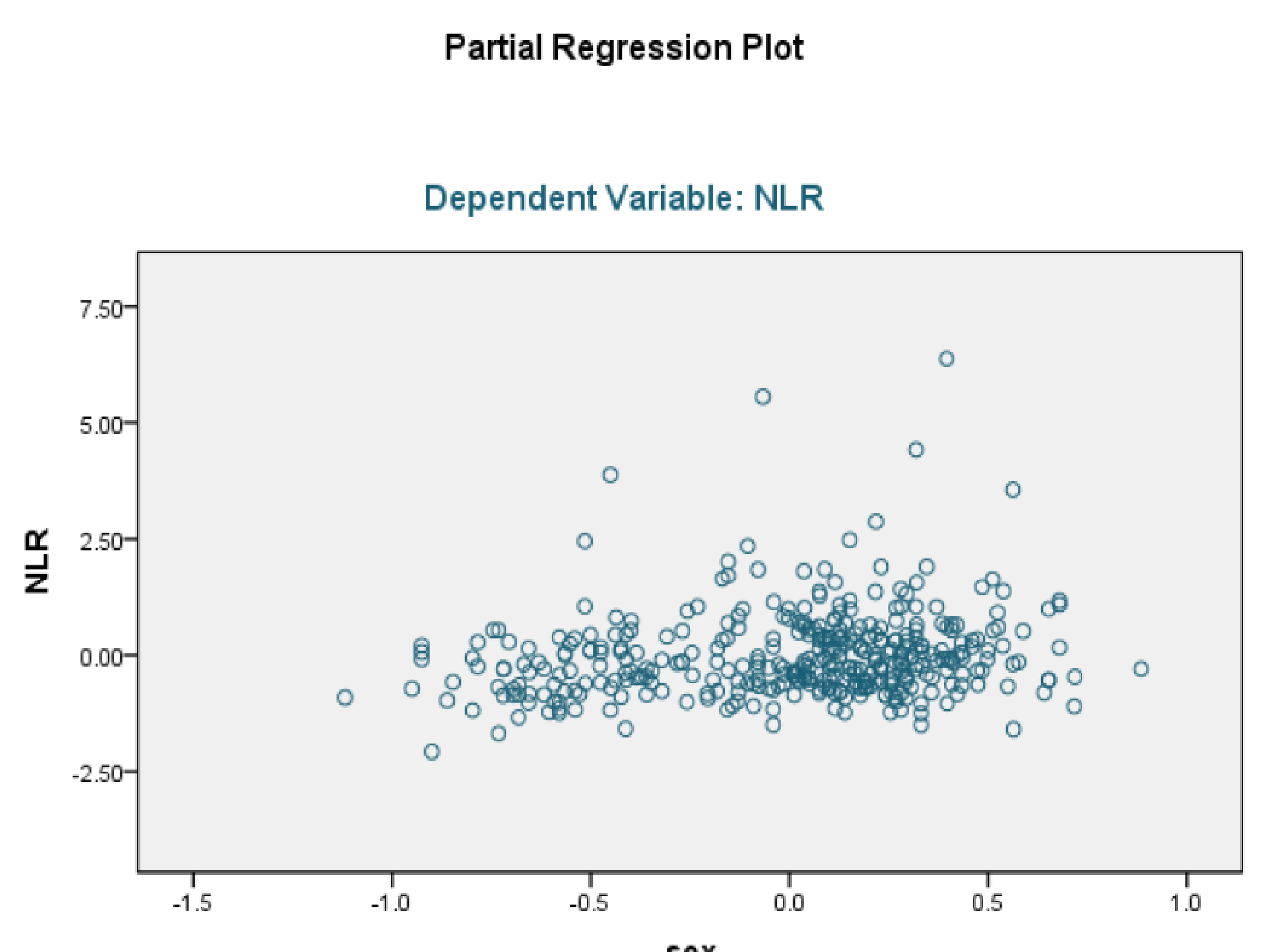

Fig. 1, Relationship between NLR and Gender

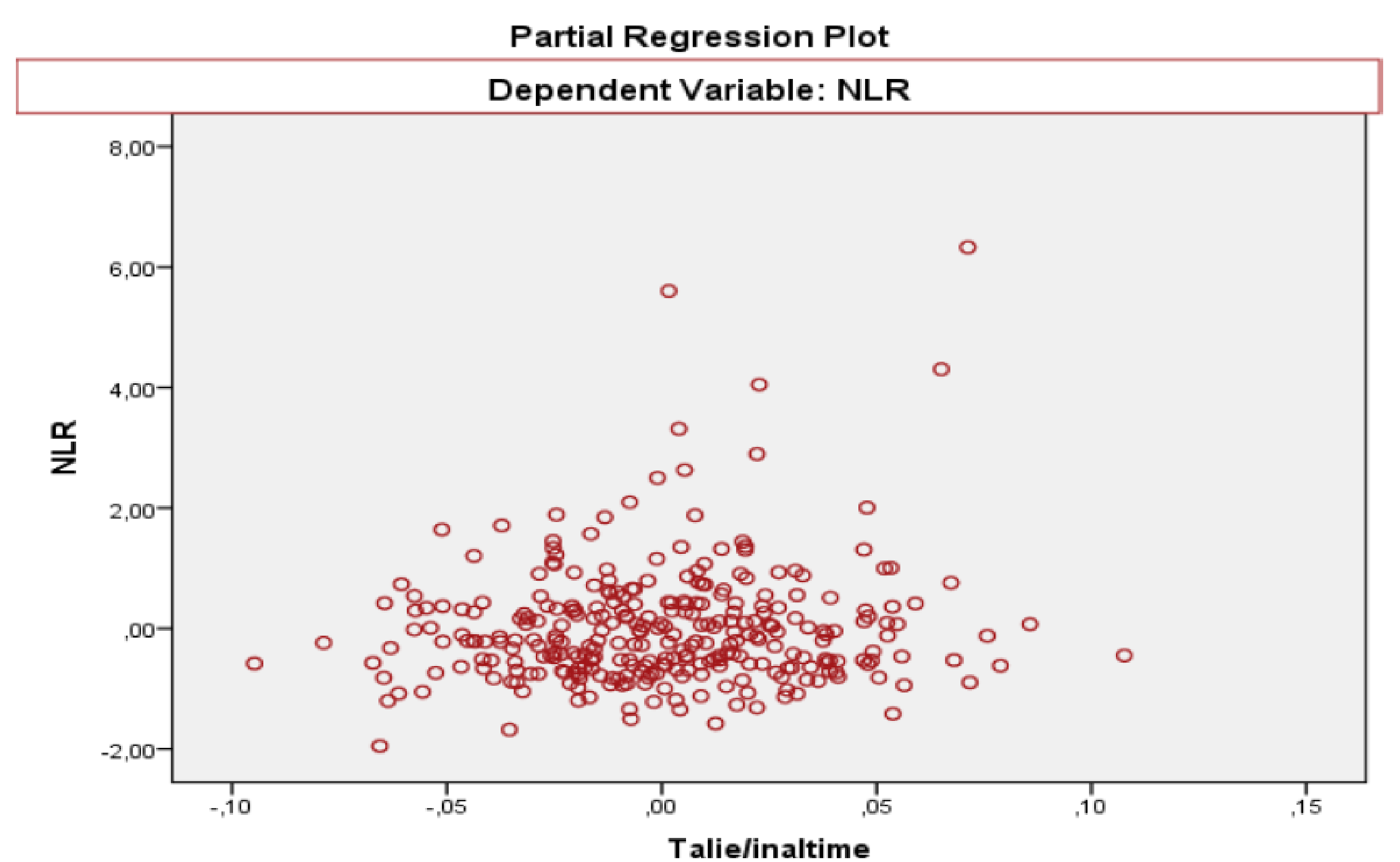

Fig. 2, Relationship between NLR and Waist/Height ratio

\section{Results}

NLR was significantly higher in women (2.33 \pm 0.95 versus $2.09 \pm 0.87, \mathrm{p}<0.05)$ and in non-smokers $(2.35 \pm 1.01$ versus $2.14 \pm 0.81, \mathrm{p}<0.05)$ After adjusting for gender and smoking status, NLR positively correlated with BMI $(r=0.132, p<0.01)$ waist circumference $(r=0.225, p<0.001)$ and waist/height ratio $(r=0.203, p<0.001)$, as well as with systolic blood pressure $(r=0.136, p<0.05)$, serum $C$ reactive protein $(r=0.170, p<0.001)$, HOMA-IR $(r=0.121, p<0.05)$ and serum leptin level $(r=0.172, p<0.01)$. In a linear regression analysis, with NLR as dependent variable and factors previously shown to significantly correlate to its level as independent variables, gender, BMI and systolic blood pressure remained independently associated with NLR.

\section{Conclusions}

Gender, systolic blood pressure and adiposity level are independent determinants of NLR in severely obese patients. Further studies are needed to determine the association of this parameter with obesity complications and prognosis. 